\title{
Allergie begreifen und bekämpfen
}

\author{
In der kleinen Kurstadt Bad Lippspringe ist 1991 ein Projekt der \\ besonderen Art entstanden: das Allergie-Dokumentations- und \\ Informationszentrum, kurz ADIZ. Daz Zentrum hat es sich zur Auf- \\ gabe gemacht, die Öffentlichkeit über Allergien zu informieren \\ und so auch zur Prävention beizutragen.
}

\begin{abstract}
C etreu dem Motto „Je mehr Sie über Ihre Krankheit wissen, desto weniger leiden Sie darunter" haben sich die Initiatoren des ADIZ unter Federführung von Prof. Dr. Karl-Christian Bergmann einiges einfallen lassen, um den Besuchern das Thema Allergie nahe zu bringen.
\end{abstract}

\section{Interaktive Einblicke in die Historie}

Das Zentrum bietet zunächst ein spannendes Museum mit Erlebniswert. Es gewährt Einblicke in die Geschichte der Allergologie und vermittelt Hintergrundwissen zu Entstehung, Diagnostik und Therapie allergischer Erkrankungen. Da mag es manchen überraschen, dass vermutlich schon die Jäger der Steinzeit unter Heuschnupfen litten. Viele Exponate kann der Besucher im wahrsten Sinne des Wortes „be-greifen“, zahlreiche interaktive Elemente machen Kindern viel Spaß und erleichtern Erwachsenen den Zugang zum Thema.

Schaustück des Museums ist eine in Deutschland einzigartige Sammlung alter Inhalationsgeräte. Eines der ältesten Geräte ist ein Pari-Inhalator aus Keramik

\section{ADIZ real und virtuell}

Das Allergie-Dokumentations- und Informationszentrum (ADIZ), Im Prinzenpalais, Burgstraße, Bad Lippspringe, ist geöffnet von Montag bis Freitag von 10.00-12.00 Uhr und von 14.00-16.00 Uhr sowie am Sonntag von 15.00-17.00 Uhr. Auch der virtuelle Besuch des ADIZ unter www.adiz.de lohnt sich.

Über das Schulungsprogramm des ADIZ informiert ebenfalls die InternetSeite oder das Sekretariat unter Tel. (0 52 52) 95-45 02. aus dem Jahr 1940. Kurios, aber durchaus mit Erkenntniswert ist eine Studie, die das ADIZ gemeinsam mit der Universität Groningen gerade durchgeführt hat. Die niederländischen Forscher untersuchten mithilfe der Laserbeugung, ob die im Museum gezeigten Inhalationsgeräte tatsächlich schon ein lungengängiges Partikelspektrum erzeugen konnten. Das Ergebnis bescheinigt den bis zu 70 Jahre alten Veteranen volle Funktionalität. Die von den Groninger Forschern entwickelte Technik kann übrigens auch für moderne Inhalatoren eingesetzt werden, um valide Daten zu deren Wirksamkeit zu erhalten.

\section{Lernen und Lustwandeln}

Doch das Museum bietet nicht nur den Blick zurück. Es werden auch auf anschauliche und interaktive Weise die Ursachen sowie die Präventions- und Behandlungsmöglichkeiten verschiedener allergischer Erkrankungen vermittelt. Darüber hinaus erhalten Interessierte und Betroffene im ADIZ umfangreiches Informationsmaterial und können in Schulungen und Vorträgen lernen, besser mit ihrer Krankheit umzugehen. Auch für medizinisches Fachpersonal gibt es Möglichkeiten zu Weiterbildung.

Direkt vor den Türen des ADIZ, auf den Terrassen unterhalb des Prinzenpalais, laden außerdem die allergologischen Gärten zu einem Besuch ein. Die Anlage wurde im Rahmen der EXPO 2000 mit über 250 verschiedenen allergieauslösenden Pflanzenarten bepflanzt. Diese wachsen verteilt auf sechs verschiedene Themengärten - von Wiese über Wald bis hin zu Gartenpflanzen. Neben der floralen Schönheit lernen Besucher hier die Pflanzen erkennen, die bei ihnen Aller-

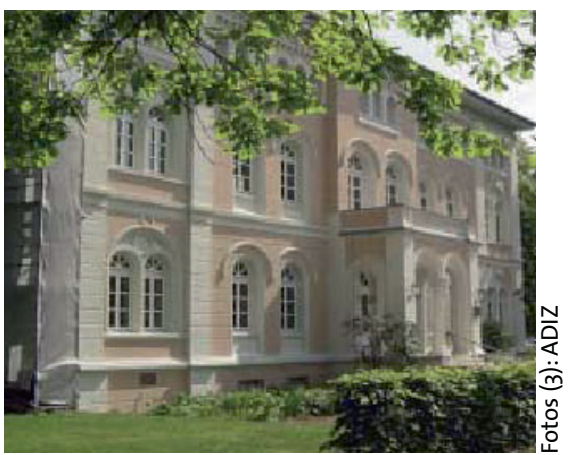

Hochherrschaftliche Adresse: Das ADIZ residiert im Bad Lippspringer Prinzenpalais.

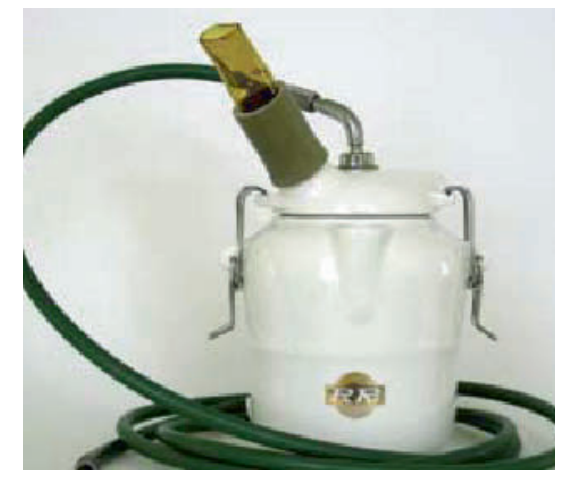

Dieses mit elektrischem Strom betriebene PARI-Dampfaerosolgerät aus dem Jahr 1940 konnte bereits ein lungengängiges Aerosol erzeugen.

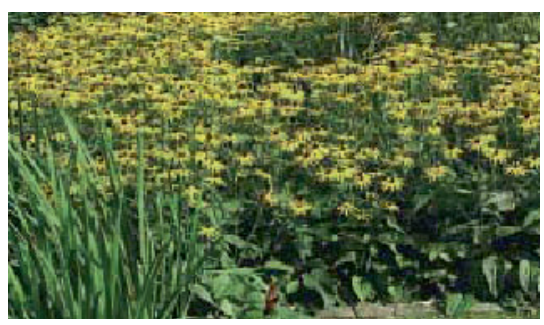

In den allergologischen Gärten wachsen über 250 allergieauslösende Pflanzen.

gien auslösen, und können sie so besser vermeiden.

Das ADIZ hat in den wenigen Jahren seines Bestehens einen hohen Stellenwert nicht nur in der Fachwelt, sondern auch bei Betroffenen aus ganz Deutschland und dem benachbarten Ausland erworben. Dies hat auch das Ministerium für Arbeit, Gesundheit und Soziales des Landes Nordrhein-Westfalen erkannt und das ADIZ 1999 als gesundheitsfördernde Institution anerkannt.

Dr. Daniela Neumann 